\title{
Rotation velocities of white dwarfs determined from the Ca II K line ${ }^{\star}$
}

\author{
L. Berger ${ }^{1}$, D. Koester ${ }^{2}$, R. Napiwotzki ${ }^{3}$, I. N. Reid ${ }^{4}$, and B. Zuckerman ${ }^{5}$ \\ ${ }^{1}$ Institut für Experimentelle und Angewandte Physik, University of Kiel, 24098 Kiel, Germany \\ e-mail: berger@physik.uni-kiel.de \\ 2 Institut für Theoretische Physik und Astrophysik, University of Kiel, 24098 Kiel, Germany \\ 3 Centre for Astrophysics Research, STRI, University of Hertfordshire, Hatfield AL10 9AB, UK \\ 4 Space Telescope Science Institute, 3700 San Martin Drive, Baltimore, MD 21218, USA \\ 5 Department of Physics \& Astronomy and Center for Astrobiology, UCLA, Los Angeles, CA 90095-1562, USA
}

Received 29 April 2005 / Accepted 22 August 2005

\section{ABSTRACT}

We determine projected rotation velocities $v \sin i$ in DAZ white dwarfs, for the first time using the rotational broadening of the Ca II K line. The results confirm previous findings that white dwarfs are very slow rotators, and set even more stringent upper limits of typically less than $10 \mathrm{~km} \mathrm{~s}^{-1}$. The few exceptions include 3 stars known or suspected to be variable ZZ Ceti stars, where the line broadening is very likely not due to rotation. The results demonstrate that the angular momentum of the core cannot be preserved completely between main sequence and final stage.

Key words. stars: white dwarfs - stars: rotation

\section{Introduction}

The last years have seen a new generation of stellar evolution models emerging, which try to incorporate the influence of rotation on internal structure and evolution (see e.g. Meynet \& Maeder 1997; Langer et al. 1998; Langer 1998; Heger et al. 2000, and later papers of these groups). These studies have been motivated by the realization that stellar surface abundances in many areas of the Hertzsprung-Russell diagram require mixing processes in addition to convection. The effects of rotation in massive stars are described in detail in Maeder \& Meynet (2000), which gives also many relevant references. In the calculation of rotating stellar models inevitably the question of angular momentum transport arises, which cannot yet be described self-consistently from first principles. Ordinary molecular viscosity is much too small, and other mechanisms have to be invoked, e.g. turbulence created through shear instability (Spiegel \& Zahn 1970; Zahn 1974, 1975, 1992), generation of internal gravity waves (Talon et al. 2002; Talon \& Charbonnel 2003, 2004), or magnetic torques (Spruit 1998).

In this situation the observation of white dwarf rotation can provide some constraints since it tells us the remaining angular momentum of the typical $0.6 M_{\odot}$ interior core of the progenitor at the end of stellar evolution. If the total angular momentum

* Based in part on data obtained at the Paranal Observatory of the European Southern Observatory for programs 165.H-0588 and 167.D-0407. of the core is preserved one would expect equatorial velocities in the range of $15 \mathrm{~km} \mathrm{~s}^{-1}$ for solar type slow-rotating progenitors to $2000 \mathrm{~km} \mathrm{~s}^{-1}$ for a fast-rotating A0 star, corresponding to rotation periods from $1 \mathrm{~h}$ down to a few minutes (a number useful to remember is that for a $0.6 M_{\odot}$ white dwarf an equatorial velocity of $15 \mathrm{~km} \mathrm{~s}^{-1}$ corresponds to a $1 \mathrm{~h}$ rotation period).

The only detailed calculation with a sophisticated treatment of angular momentum transfer, which makes a specific prediction for the final white dwarf rotational velocity is the work of Langer et al. (1999). For the $0.8 M_{\odot}$ white dwarf resulting from the evolution of a rotating $3 M_{\odot}$ model they expect an equatorial rotation of $28 \mathrm{~km} \mathrm{~s}^{-1}$.

On the other hand - assuming the core to remain strongly coupled to the outer expanding envelope in the giant stage - the rotation of the final white dwarf should be extremely slow, with periods in the range 30 to 1000 years (Spruit 1998). Rotation periods of hours to days have in a few cases been determined from the rotational splitting of pulsation frequencies; a list is given in Spruit (1998), to which could be added a period of $13 \mathrm{hrs}$ for L19-2 and $58 \mathrm{hrs}$ for GD165 (Bradley 2001). Periods around one day have also been measured from variable polarization (Schmidt \& Norsworthy 1991; Schmidt \& Smith 1995), but for some of these magnetic white dwarfs no variation has been observed, implying rotation periods of at least a century.

The great majority of rotation data for white dwarfs have been determined spectroscopically, exclusively using the sharp NLTE core of the $\mathrm{H} \alpha$ line in DA white dwarfs 
(Pilachowski \& Milkey 1984, 1987; Milkey \& Pilachowski 1985; Koester \& Herrero 1988; Heber et al. 1997; Koester et al. 1998; Karl et al. 2005). The sample now contains about 50 stars with determined $v \sin i$. A few of these objects show a line broadening suggesting velocities of a few dozen $\mathrm{km} \mathrm{s}^{-1}$, but in many cases the unsatisfactory line fits indicate that the reason is not rotation but magnetic splitting or unknown, as in the case of the ZZ Ceti stars (Koester et al. 1998). In the majority of objects only upper limits can be determined which typically range from 10 to $30 \mathrm{~km} \mathrm{~s}^{-1}$ and are thus marginally compatible with the result of Langer et al. (1999). While this does not rule out that most white dwarfs could be extremely slow rotators, Spruit (1998), taking all available empirical evidence and following his idea of strong core-envelope coupling felt a need for mechanisms, which would accelerate the white dwarfs again to the observed values after the efficient loss of angular momentum in the giant phase.

There is thus clearly a strong motivation to determine more stringent upper limits using the spectroscopic method. This has become possible after the detection of a large number of DAZ objects found in the studies of Zuckerman \& Reid (1998); Zuckerman et al. (2003) and as a byproduct of the search for Supernova Ia progenitors (Napiwotzki et al. 2003, 2001; Koester et al. 2005). The Ca II K line in these hydrogen-rich white dwarfs is deep and quite narrow and should be useful for measuring much lower rotation velocities than is possible with $\mathrm{H} \alpha$. In this paper we report the results of such a study.

\section{Observations}

The sample consisted of spectra for 38 single cool DA white dwarfs coming from two different sources. The first is the SPY survey (ESO Supernova Ia Progenitor Survey) for doubledegenerates. In the search for variable radial velocities a large number of high-resolution spectra are obtained with the UVES spectrograph at the ESO VLT Paranal Observatory, which are useful also for many other projects. Rollenhagen (2004) and Koester et al. (2005) used this sample to identify hydrogen-rich white dwarfs with CaII lines and those objects form the first part of our present sample (excluding those where the lines are interstellar). Details of the observations and the reduction procedures are described in Napiwotzki et al. (2003) and Koester et al. (2001); we therefore repeat here only the facts relevant for this project. The observations used a wide slit of 2 '. 1 , because the SPY was conducted as a filler project for bad weather conditions. This is important since the nominal resolution with this slit is 18500 corresponding to $0.21 \AA$ at the Ca II K line, however, the actual resolution can be higher if the seeing disk is smaller than the slit. The actual seeing during the SPY observations typically varied between 1 .'0 to 1 '. 5 .

The second source of sample objects are Keck observations of DAs in a search for DAZs with metals. The observations are described in Zuckerman \& Reid (1998) and Zuckerman et al. (2003). Here the nominal resolution with a slit width of 1 '. 15 is 34000 , corresponding to $0.12 \AA$ at Ca II K, but also in this case the actual resolution might be slightly higher at times of excellent seeing.
Table 1. Atmospheric parameters $T_{\text {eff }}$ in $\mathrm{K}$, surface gravity $\log g$, and $\mathrm{Ca}$ abundance for the sample. $[\mathrm{Ca} / \mathrm{H}]$ is the logarithm of the $\mathrm{Ca} / \mathrm{H}$ abundance ratio by numbers. Column Ref gives the source of the parameters with $\mathrm{Z}=$ Zuckerman et al. (2003), $\mathrm{R}=$ Rollenhagen (2004) and Koester et al. (2005). Columns $\mathrm{K}$ and $\mathrm{S}$ give the number of available spectra from the Keck or the SPY samples. Please note that the Ca abundance for HS 0047+1903 in Koester et al. (2005) was based on an erroneous equivalent width. The correct abundance is given in Table 2.

\begin{tabular}{lrrrrrr}
\hline \hline Object & $T_{\text {eff }}$ & $\log g$ & {$[\mathrm{Ca} / \mathrm{H}]$} & $\mathrm{Ref}$ & $\mathrm{K}$ & $\mathrm{S}$ \\
\hline WD 0032-175 & 9235 & 8.0 & -10.2 & $\mathrm{Z}$ & 1 & \\
HS 0047+1903 & 16600 & 7.8 & & $\mathrm{R}$ & & 1 \\
HE 0106-3253 & 15700 & 8.0 & -6.4 & $\mathrm{R}$ & & 2 \\
WD 0208+296 & 7201 & 7.9 & -8.8 & $\mathrm{Z}$ & 2 & \\
WD 0235+064 & 11420 & 7.9 & -9.0 & $\mathrm{Z}$ & 1 & \\
WD 0243-026 & 6798 & 8.2 & -9.9 & $\mathrm{Z}$ & 1 & 2 \\
WD 0245+541 & 5190 & 8.2 & -12.7 & $\mathrm{Z}$ & 1 & \\
HS 0307+0746 & 10200 & 8.1 & -7.6 & $\mathrm{R}$ & & 1 \\
WD 0408-041 & 14400 & 7.8 & -7.1 & $\mathrm{R}$ & & 2 \\
WD 0543+579 & 8142 & 8.0 & -10.3 & $\mathrm{Z}$ & 3 & \\
WD 0846+346 & 7373 & 8.0 & -9.4 & $\mathrm{Z}$ & 2 & \\
WD 1015+161 & 19300 & 7.9 & -6.3 & $\mathrm{R}$ & & 2 \\
WD 1102-183 & 8026 & 8.0 & -10.4 & $\mathrm{Z}$ & 1 & \\
WD 1116+026 & 12200 & 7.9 & -7.3 & $\mathrm{R}$ & & 2 \\
WD 1124-293 & 9680 & 8.0 & -8.5 & $\mathrm{Z}$ & 2 & 2 \\
WD 1150-153 & 12800 & 7.8 & -6.7 & $\mathrm{R}$ & & 2 \\
WD 1202-232 & 8619 & 8.0 & -9.8 & $\mathrm{Z}$ & 2 & 2 \\
WD 1204-136 & 11468 & 8.0 & -7.7 & $\mathrm{Z}$ & 1 & 2 \\
WD 1208+576 & 5830 & 7.9 & -11.0 & $\mathrm{Z}$ & 1 & \\
HE 1225+0038 & 9400 & 8.1 & -9.7 & $\mathrm{R}$ & & 3 \\
WD 1257+278 & 8481 & 7.9 & -8.1 & $\mathrm{Z}$ & 2 & \\
HE 1315-1105 & 9400 & 8.4 & -9.2 & $\mathrm{R}$ & & 2 \\
WD 1337+705 & 20435 & 7.9 & -6.7 & $\mathrm{Z}$ & 3 & \\
WD 1344+106 & 6945 & 8.0 & -11.1 & $\mathrm{Z}$ & 2 & \\
WD 1407+425 & 9856 & 8.0 & -9.9 & $\mathrm{Z}$ & 1 & \\
WD 1455+298 & 7366 & 7.6 & -9.3 & $\mathrm{Z}$ & 2 & \\
WD 1457-086 & 20400 & 8.0 & -6.3 & $\mathrm{R}$ & & 2 \\
WD 1614+160 & 17400 & 7.8 & -7.2 & $\mathrm{R}$ & & 2 \\
WD 1633+433 & 6569 & 8.1 & -8.6 & $\mathrm{Z}$ & 2 & \\
WD 1821-131 & 7029 & 8.4 & -10.7 & $\mathrm{Z}$ & 1 & \\
WD 1826-045 & 9480 & 7.9 & -8.8 & $\mathrm{Z}$ & 1 & 1 \\
WD 1858+393 & 9470 & 8.0 & -7.8 & $\mathrm{Z}$ & 2 & \\
WD 2115-560 & 9700 & 8.1 & -7.6 & $\mathrm{R}$ & & 1 \\
HS 2132+0941 & 13200 & 7.7 & -7.7 & $\mathrm{R}$ & & 2 \\
WD 2149+021 & 17300 & 7.9 & -7.6 & $\mathrm{R}$ & & 2 \\
HE 2221-1630 & 10100 & 8.2 & -7.6 & $\mathrm{R}$ & & 2 \\
HS 2229+2335 & 18600 & 7.9 & -6.3 & $\mathrm{R}$ & & 2 \\
WD 2326+049 & 11600 & 8.1 & -6.9 & $\mathrm{Z}$ & 5 & 2 \\
\hline & & & & & &
\end{tabular}

For most objects more than one spectrum is available, for some even spectra from both surveys. Atmospheric parameters $T_{\text {eff }}, \log g$, and Ca abundances for the sample are given in Koester et al. (2005) and Zuckerman et al. (2003) (which also show some representative spectra) and are collected in Table 1. For our analysis the spectra were shifted slightly to bring the $\mathrm{K}$ line in agreement with the laboratory wavelength and then normalized to a continuum of 1.0 with a straight line fitted to the continuum approximately $5 \AA$ on both sides of the line center. 


\section{Determination of rotation velocities}

Rotation velocities were determined using theoretical model atmospheres with spectral lines broadened by a rotational broadening function (see e.g. Unsöld 1968). Our model grid is calculated assuming LTE (local thermodynamic equilibrium) with methods and input physics described in Finley et al. (1997), Koester \& Wolff (2000), and Homeier et al. (1998). The Ca II lines have the advantage compared to the Balmer lines - the only lines used in the past for spectroscopic determinations in white dwarfs - that the central Doppler core from thermal broadening is much narrower because of the higher atomic weight.

On the other hand, the Balmer lines originate from the dominant element in DAs and therefore only two parameters, $T_{\text {eff }}$ and $\log g$, are needed to describe the fitting model. In the case of the $\mathrm{K}$ line an additional parameter is the $\mathrm{Ca}$ abundance. The results given in Zuckerman et al. (2003) and Koester et al. (2005) have been determined using interpolations in precalculated tables of equivalent widths. In the calculations of the theoretical equivalent widths a fixed interval was assumed, and no attempt was made to match this exactly in the observed spectra; moreover, the spacing of the grid of abundances was rather coarse. It is therefore not surprising that calculated spectra for the "exact" atmospheric parameters using these $\mathrm{Ca}$ abundances did not always match the spectra perfectly. We have then changed the $\mathrm{Ca}$ abundance (typically less than a factor of two) until the EW of the observation agreed exactly with that of the theoretical model. This is obviously a prerequisite for the determination of rotational broadening of the profile.

The standard rotational broadening profile uses two free parameters, the limb darkening coefficient $\beta$ and the projected equatorial rotation velocity $v \sin i$. Here $i$ is the inclination of the rotation axis against the line-of-sight, ranging from 0 to 90 degrees (note that only this combination can be determined with the spectroscopic method). Our theoretical models show that $\beta$ varies from $\approx 0.2$ at the center of the $\mathrm{K}$ line to $\approx 1.2$ in the far wing. Since the parameter has almost no influence on the result we have continued to use $\beta=0.15$ which is used by all authors for $\mathrm{H} \alpha$. The best fit value for $v \sin i$ and the errors were then determined with a $\chi^{2}$ fitting routine very similar to that used in Koester et al. (1998). Figure 1 shows the observed line in WD 1826-045 as a typical example together with theoretical models with different rotational velocities applied. The figure demonstrates that rotational velocities of a few $\mathrm{km} \mathrm{s}^{-1}$ can easily be measured from the $\mathrm{Ca}$ line.

\subsection{A remark on the standard spectroscopic method}

Assuming that the intensity of the radiation field at the surface of a star depends only on wavelength $\lambda$ and the angle $\theta$ against the normal, the observational quantity for a non-rotating star is the disk-averaged intensity

$\bar{I}=\frac{1}{\pi} \int_{-1}^{+1} \int_{-\sqrt{1-x^{2}}}^{+\sqrt{1-x^{2}}} I(\mu, \lambda) \mathrm{d} x \mathrm{~d} y$

where $\mu=\cos \theta$, and $x, y$ are Cartesian coordinates on the visible surface of the star in units of the stellar radius $R$.

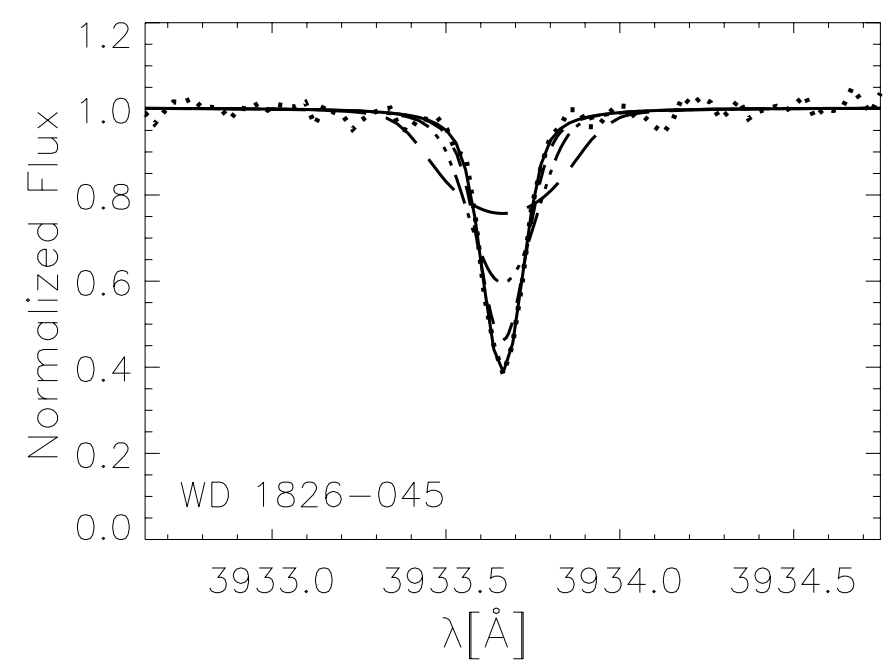

Fig. 1. Ca II K line profile in WD 1826-045. Dotted: observed profile; continuous: non-rotating model after convolution with instrumental profile (falls on top of the observation in the line center!); dashed and dot dashed with decreasing line depths: rotating models with $v \sin i=$ $5,10,20 \mathrm{~km} \mathrm{~s}^{-1}$.

In the case of a rotating star we have in addition a Doppler shift, which depends on the position on the disk

$\bar{I}=\frac{1}{\pi} \int_{-1}^{+1} \int_{+\sqrt{1-x^{2}}}^{-\sqrt{1-x^{2}}} I(\mu, \lambda-\Delta \lambda(x, y)) \mathrm{d} x \mathrm{~d} y$.

If the $y$-axis is chosen parallel to the rotation axis, $\Delta \lambda$ will depend on $x$ only. This integral can easily be evaluated numerically if theoretical models are available for the intensity as a function of $\mu$. It is common practice, however, to proceed with an analytic evaluation by assuming a limb-darkening law

$I(\mu)=I_{\mathrm{c}}((1-\epsilon)+\epsilon \mu)$

with central intensity $I_{\mathrm{c}}$. This law can also be written relative to the intensity at the limb $I_{1}$

$I(\mu)=I_{1}(1+\beta \mu)$

with the limb-darkening coefficient $\beta=\epsilon /(1-\epsilon)$ used above. Introducing this into the integration over the disk, with $\mu$ a function of position $x, y$, the integration can be transformed into a convolution of the disk-averaged intensity for a non-rotating star

$\bar{I}_{\text {rot }}=\int_{-1}^{+1} G(x) \bar{I}\left(\lambda-x \Delta \lambda_{l}\right) \mathrm{d} x$

with the maximum shift at the limb $\Delta \lambda_{1}$, and

$G(x)=\frac{4(1-\epsilon)+\epsilon \sqrt{1-x^{2}}+\pi \epsilon\left(1-x^{2}\right)}{2 \pi(1-\epsilon / 3)}$

(see e.g. Unsöld 1968). This is a large simplification, since only the theoretical disk-averaged intensity (also called the astrophysical flux) needs to be calculated, which is always needed 
for the analysis of spectra, rotating or not. However, the derivation makes the assumption that the limb darkening law is independent of wavelength, in particular that the coefficients $\epsilon$ or $\beta$ are constant over the line profile. This is clearly not the case for the Ca II $\mathrm{K}$ line as mentioned above and it is also not true for $\mathrm{H} \alpha$. We have therefore studied the effect of this assumption by comparing the exact direct integration with angle-dependent intensities with the simplified method using the convolution technique. While this has been studied before in the literature for other types of stars it has to our knowledge never been tested for white dwarf spectra.

The result of this test is (Pruin 2005) that small differences between the two methods exist in the line cores, but that the error made when using the simple convolution method is much smaller than $1 \mathrm{~km} \mathrm{~s}^{-1}$, and thus negligible in our study.

\subsection{Observed and theoretical line profiles}

When comparing the observed and theoretical profiles after taking into account instrumental broadening with the nominal resolution in the latter, we obtained nearly perfect fits for about half of the objects without rotational broadening, implying zero or at least extremely low rotation. In the other half of objects the observed line profile had a deeper core than our "unrotated" models. Since rotational broadening can only decrease the central depths, we could thus not obtain a fit.

What could be the reason for this discrepancy? The line profiles are of course influenced by the reduction process, e.g. the subtraction of sky background, to mention just one step which could affect especially the line cores. Indeed, when we have several spectra of the same object, there are sometimes differences in equivalent width and also line depth. Another possibility is on the modeling side: the largest discrepancies (in seven objects) occur for very deep lines, where the core is saturated. In these cases the line core originates in the outermost layers of the atmosphere and it is quite possible that NLTE effects (not included in our models) will make the model lines deeper, as is well known for $\mathrm{H} \alpha$.

We believe, however, that in most objects the reason is that the actual resolution of the observation was better than nominal. This is supported by the finding that in several spectra of the same stars the equivalent width is constant but the line profile changes, with the line being deeper and narrower in some of the spectra. This strongly indicates a changing actual resolution for periods with better seeing.

Unfortunately we do not know the real resolution for the observation. In order to be able to nevertheless extract some information on the rotation velocities we have therefore increased the resolution (narrowed the instrumental profile) until our models achieved a good fit without rotation. We have then used our $\chi^{2}$ routine to determine $v$ sin $i$. Obviously the result must be compatible with zero, but we nevertheless obtain a reasonable upper limit from the errors provided by the fit. The results obtained for 31 objects for which this procedure led to a good fit with the nominal or within a plausible range of resolutions (see below) are shown in Table 2. A number in the
Table 2. Rotational velocities. When several spectra of the same object are of comparable quality, the results do not differ significantly and we therefore give only the result from the best spectrum. The only exception is WD2326+049, a known ZZ Ceti variable. $[\mathrm{Ca} / \mathrm{H}]$ is the $\mathrm{Ca}$ abundance as determined from fitting the line profile, which is sometimes slightly different from that in the original source (see Table 1; the corrected EW for HS $0047+1903$ used for the abundance in this table is $77 \mathrm{~m} \AA$ ). $v_{2}=v \sin i$ is the rotation velocity determined using either the nominal resolution of the spectrographs (34000 for Keck, 18500 for SPY), or a slightly higher resolution if the line depth cannot be reproduced at nominal resolution. In the latter case the values for the resolution are given in column Res. Errors are $1 \sigma$ errors. $v_{1}=(v \sin i)_{\max }$ is an upper limit using the optimum resolution as explained in the text.

\begin{tabular}{|c|c|c|c|c|}
\hline$\overline{\text { Object }}$ & $\overline{[\mathrm{Ca} / \mathrm{H}]}$ & $\overline{v_{1}}$ & $\overline{\overline{v_{2}}}$ & $\overline{\overline{R e s}}$ \\
\hline WD 0032-175 & -10.5 & 3.8 & $0.0 \pm 2.8$ & 46300 \\
\hline HS $0047+1903$ & -6.1 & 11.6 & $9.9 \pm 6.1$ & \\
\hline HE $0106-3253$ & -5.8 & 6.1 & $0.0 \pm 4.9$ & 28100 \\
\hline WD $0235+064$ & -9.2 & 5.1 & $0.0 \pm 4.0$ & 39300 \\
\hline WD $0243-026$ & -9.8 & 4.7 & $2.5 \pm 4.2$ & 32800 \\
\hline WD $0245+541$ & -11.7 & 0.0 & $0.0 \pm 6.6$ & \\
\hline HS $0307+0746$ & -7.1 & 9.1 & $5.4 \pm 5.5$ & 24600 \\
\hline WD 0408-041 & -6.6 & 7.1 & $0.0 \pm 5.1$ & 24600 \\
\hline WD $0543+579$ & -10.5 & 5.0 & $3.4 \pm 1.7$ & 46300 \\
\hline WD 1015+161 & -5.9 & 10.1 & $2.4 \pm 4.7$ & 20700 \\
\hline WD 1102-183 & -10.3 & 8.1 & $5.9 \pm 1.9$ & \\
\hline WD $1116+026$ & -6.5 & 11.4 & $3.6 \pm 3.8$ & \\
\hline WD 1124-293 & -8.2 & 5.8 & $0.0 \pm 2.2$ & \\
\hline WD 1150-153 & -6.0 & 14.3 & $9.5 \pm 5.4$ & \\
\hline WD 1202-232 & -9.8 & 5.8 & $0.0 \pm 0.9$ & \\
\hline WD 1204-136 & -7.2 & 11.1 & $9.7 \pm 0.8$ & \\
\hline WD $1208+576$ & -10.8 & 8.8 & $6.5 \pm 1.9$ & \\
\hline HE $1225+0038$ & -9.7 & 9.4 & $0.0 \pm 7.0$ & 21300 \\
\hline HE $1315-1105$ & -9.4 & 6.7 & $1.5 \pm 4.8$ & 27100 \\
\hline WD $1337+705$ & -6.7 & 4.4 & $0.0 \pm 1.2$ & 41400 \\
\hline WD 1344+106 & -11.3 & 7.2 & $4.0 \pm 2.5$ & \\
\hline WD $1407+425$ & -9.8 & 8.9 & $6.7 \pm 1.5$ & \\
\hline WD 1457-086 & -6.1 & 9.6 & $0.0 \pm 8.5$ & 21900 \\
\hline WD $1614+160$ & -7.2 & 2.2 & $0.4 \pm 4.9$ & 35750 \\
\hline WD $1821-131$ & -10.9 & 18.0 & $15.8 \pm 4.6$ & \\
\hline WD 1826-045 & -8.6 & 3.1 & $0.0 \pm 1.3$ & 49200 \\
\hline HS $2132+0941$ & -7.1 & 8.5 & $0.0 \pm 8.3$ & 21260 \\
\hline WD 2149+021 & -7.7 & 10.7 & $0.0 \pm 10.3$ & 20700 \\
\hline HE $2221-1630$ & -7.2 & 4.2 & $2.1 \pm 4.0$ & 34200 \\
\hline HS $2229+2335$ & -5.9 & 6.5 & $0.0 \pm 5.2$ & 27100 \\
\hline \multirow[t]{7}{*}{ WD $2326+049$} & -6.4 & 20.5 & $19.8 \pm 0.5$ & \\
\hline & -6.3 & 15.7 & $14.6 \pm 0.5$ & \\
\hline & -6.3 & 28.2 & $27.6 \pm 0.4$ & \\
\hline & -6.3 & 26.9 & $26.2 \pm 0.3$ & \\
\hline & -6.3 & 28.4 & $28.0 \pm 0.4$ & \\
\hline & -6.4 & 15.3 & $10.7 \pm 2.1$ & \\
\hline & -6.4 & 22.5 & $20.0 \pm 0.9$ & \\
\hline
\end{tabular}

resolution column indicates the higher than nominal resolution used in the fit.

There is of course some arbitrariness involved with this method, since the resolution could actually be even higher, and could also have been higher in the cases where our zerorotation models had lines deep enough. In order to analyze the uncertainty we have assumed a maximum plausible resolution 


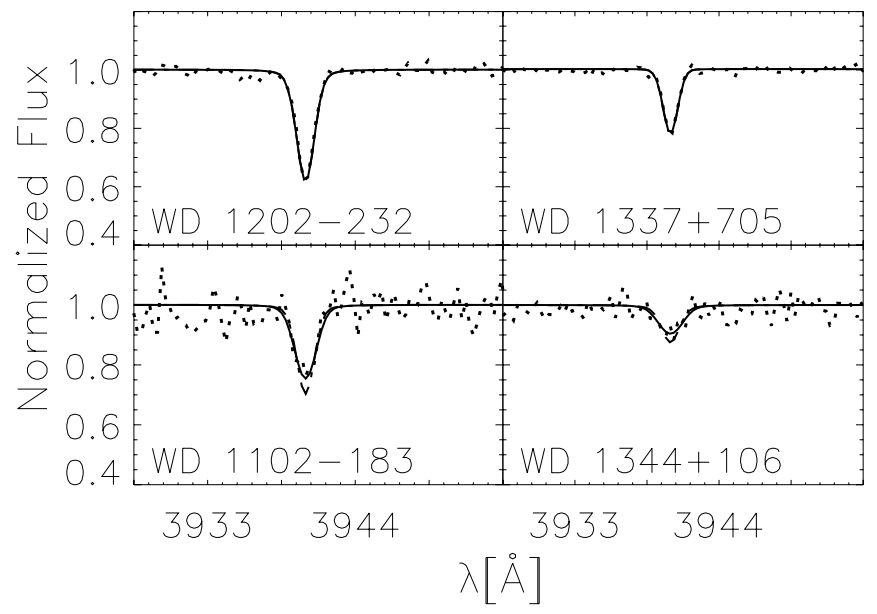

Fig. 2. Typical examples for the determination of rotational velocities for the Ca II K line, ranging from some of the best spectra to marginal. Dotted: observed profile; dashed: non-rotating model after convolution with instrumental profile; continuous: best fit with rotation. In the two top figures the best fit is zero rotation and the models fall on top of each other.

of 39500 and 65500 , equivalent to seeing of 1.' 0 and $0 .{ }^{\prime} 6$, respectively, for SPY and Keck spectra. The column $v \sin i(\max )$ gives the results of the fitting procedure, which should be considered as very firm upper limits.

Figure 2 presents four typical examples from spectra with high to rather low signal-to-noise ratios.

\subsection{Error estimates}

We estimated the errors caused by the uncertainties of $T_{\text {eff }}$, $\log g,[\mathrm{Ca} / \mathrm{H}], \beta$, and the resolution. The summed errors from the first four quantities were of the same order of magnitude as the formal $1 \sigma$-error of the $\chi^{2}$-fitting given in Table 2 . The greatest error was caused by the uncertainty of the resolution and can be estimated by comparing the values in the column $v \sin i$ and $(v \sin i)_{\max }$.

\subsection{Objects with very deep line cores}

Even at the assumed maximum increase of the nominal resolution there remain seven objects which cannot be fitted by unrotated profiles. All of these objects have strong $\mathrm{Ca}$ lines, which are saturated in the core. In these cases we have used a Lorentz profile with zero central intensity and a width calculated to reproduce the observed EW. These profiles were then used to fit the observed profiles, resulting in firm upper limits to the rotational velocities. These results are given in Table 3 . The spectra and fits are shown in Fig. 3, where the dotted lines are the observations, the dashed lines Lorentz profiles with zero central intensity as an extreme simulation of the line profile, and the continuous lines are the "rotated" profiles.

\section{Discussion}

21 objects in Table 2 and all 7 in Table 3 are compatible with zero rotation velocities (within $1 \sigma$ errors). Even allowing for

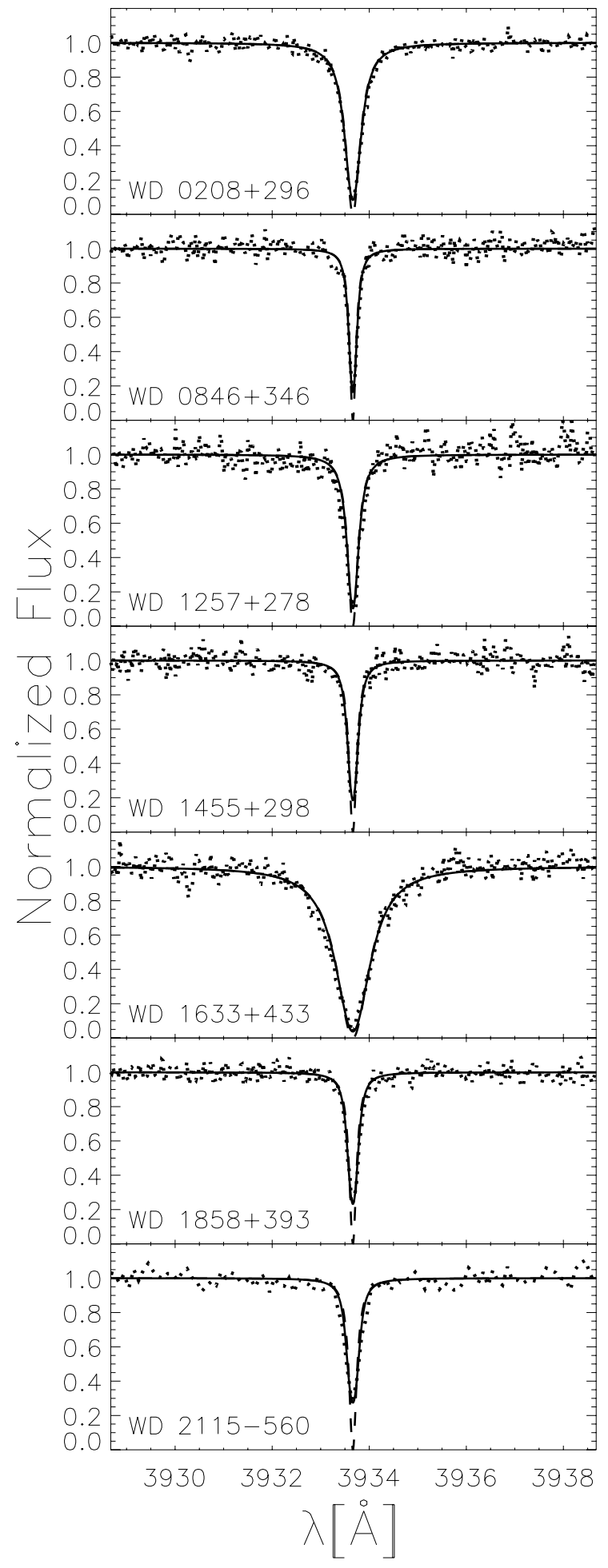

Fig. 3. Seven objects fitted with Lorentz profiles. See text.

a higher than nominal resolution, within the plausible range, gives upper limits typically smaller than $10 \mathrm{~km} \mathrm{~s}^{-1}$. This is a clear improvement compared to the spectroscopic determination from the core of $\mathrm{H} \alpha$, with many upper limits in the range 10-30 $\mathrm{km} \mathrm{s}^{-1}$ (Koester et al. 1998; Karl et al. 2005). Unfortunately we are aware of only two $\mathrm{H} \alpha$ determinations for objects of our sample, both in Koester et al. (1998). For WD 2115-560 the upper limit from $\mathrm{H} \alpha$ is $35 \mathrm{~km} \mathrm{~s}^{-1}$, while we 
Table 3. Upper limit to the rotational velocities for the objects with deeper observed line profiles than is possible with a non-rotating model. The limits were determined using a Lorentz line profile with central intensity zero and equivalent width $(E W$, in $m \AA)$ equal to the observed spectrum.

\begin{tabular}{lcrr}
\hline \hline Object & $S / N$ & $E W$ & $(v \sin i)_{\max }$ \\
\hline WD 0208+296 & 37 & 534 & 8 \\
WD 0846+346 & 24 & 245 & 6 \\
WD 1257+278 & 15 & 375 & 7 \\
WD 1455+298 & 21 & 282 & 8 \\
WD 1633+433 & 19 & 1200 & 13 \\
WD 1858+393 & 27 & 285 & 9 \\
WD 2115-560 & 24 & 292 & 11 \\
\hline
\end{tabular}

obtain $11 \mathrm{~km} \mathrm{~s}^{-1}$ from the $\mathrm{Ca}$ line. The other common object is the ZZ Ceti star WD 2326+049 (G29-38). From H $\alpha$ a velocity of $45 \pm 5 \mathrm{~km} \mathrm{~s}^{-1}$ is obtained, but the authors note that the fit is not good and the broadening very likely not due to rotation. From our Ca determination we find a range of 14-28 $\mathrm{km} \mathrm{s}^{-1}$ from different spectra.

G29-38 has been very extensively studied as a pulsator, both with photometry and spectroscopy. van Kerkwijk et al. (2000) detected periodic Doppler shifts in the line profiles with amplitudes up to $5 \mathrm{~km} \mathrm{~s}^{-1}$, which they tentatively associated with the horizontal motions of g-mode oscillations. Thompson et al. (2003) recently found Doppler shifts in G29-38 corresponding to radial velocity changes up to $16.5 \mathrm{~km} \mathrm{~s}^{-1}$. By combining a large number of spectra with close to zero Doppler shift and comparing with an average spectrum over several periods they found no significant difference in the line profiles. They concluded that pulsation cannot be the reason for the flatbottom line profiles. This conclusion may be somewhat premature, however, since the study only demonstrates that even the profile in one particular pulsation phase does not fit a rotationally broadened model spectrum. It is plausible that even with an average shift of zero velocity in that phase different parts of the stellar surface may show positive as well as negative velocity along the line-of-sight, leading to zero shift but a broadening of the profile. This can only be decided with numerical simulations of the pulsating surface including all shifts and realistic center-to-limb variations. In any case the difference between the $\mathrm{H} \alpha$ and $\mathrm{Ca}$ results and the significant variation of the $\mathrm{Ca}$ "rotation velocities" confirm convincingly that rotation is not the origin of the broadening.

WD $2326+049$ is one of the 10 objects for which we determine a non-zero rotation velocity for the best fitting model. Two others are WD 1150-153 and WD 1204-136, which according to their atmospheric parameters fall within or near the ZZ Ceti instability strip. WD 1204-136 was found to be non-variable (upper limit $0.3 \%$ ) by Bergeron et al. (2004), while WD 1150-153 to our knowledge has not been observed for variability. The latter is on our candidate list for upcoming time-resolved photometry, but in any case it seems very likely that the same mechanism - as yet unidentified - is responsible for the line shapes as in the known ZZ Ceti stars (Koester et al. 1998). Four other white dwarfs with nonzero rotation (WD $0543+579$, WD $1407+425$, WD 1344+106,
WD 1821-131) are cool and the parameters are based on photometry only. In the first two cases the parallax is not known and thus the surface gravity cannot be determined and was fixed at 8.0 (Zuckerman et al. 2003). However, even in these cases the rotation velocities are definitely very small, $<18 \mathrm{~km} \mathrm{~s}^{-1}$ at most.

The results of this paper confirm the findings of all previous studies, that white dwarfs are slow rotators, and set even more stringent limits for those objects with $\mathrm{Ca}$ lines. Typical rotation velocities are below $10 \mathrm{~km} \mathrm{~s}^{-1}$ and may even be close to zero velocity for many objects. While this could be just marginally consistent with conservative evolution for solartype slow-rotating progenitors, the result would definitely exclude conservation of angular momentum for more massive progenitors. How many of such white dwarfs could we expect in our sample?

Assuming for simplicity a unique initial-final mass relation between remnant and progenitor, we find from Weidemann (2000) that a $0.6 M_{\odot}$ white dwarf originates from a $2 M_{\odot}$ main sequence progenitor. $0.6 M_{\odot}$ (at $T_{\text {eff }}=10000 \mathrm{~K}$ ) correspond to $\log g=8.0$. Taking the numbers for the atmospheric parameters in Table 1 at face value, we have 27 objects with a $\log g$ determination (versus assuming 8.00), of which 11 have $\log g \geq 8.0$ and should have originated from fast-rotating main sequence stars more massive than $2.0 M_{\odot}$. While the exact numbers may be uncertain, there is no doubt that these results mean that angular momentum in the core cannot be preserved. These results also clearly rule out the prediction by Langer et al. (1999), which were based on stellar model calculations without magnetic fields.

While the original study of Spruit (1998) predicted very efficient angular momentum transport through the magnetic field coupling of core and outer envelope, the new quantitative formulation of magnetic field generation and magnetic field induced angular momentum transport developed in Spruit (2002) leads to a more limited angular momentum transport efficiency. First calculations for massive stars (Heger et al. 2005) including the Spruit (2002) magnetic field physics show these stars evolving far from rigid rotation. Similar results are expected for low mass stars (Langer 2005, priv. comm.), which would likely bring the predictions for the white dwarf rotation velocities in agreement with the new observations.

Our method could in principle be improved further, if that is necessary to distinguish between theoretical assumptions. The main reason for the current uncertainty is the unknown effective resolution due to rather large slits used in the spectroscopy. With a narrower slit and well controlled resolution an accuracy of $1-2 \mathrm{~km} \mathrm{~s}^{-1}$ could be reached, but the observations would need a significant amount of observing time on a very large telescope.

\section{References}

Bergeron, P., Fontaine, G., Billères, M., Boudreault, S., \& Green, E. M. 2004, ApJ, 600, 404

Bradley, P. A. 2001, ApJ, 552, 326

Finley, D. S., Koester, D., \& Basri, G. 1997, ApJ, 488, 375

Heber, U., Napiwotzki, R., \& Reid, I. N. 1997, A\&A, 323, 819 
Heger, A., Langer, N., \& Woosley, S. E. 2000, ApJ, 528, 368

Heger, A., Woosley, S. E., \& Spruit, H. C. 2005, ApJ, 626, 350

Homeier, D., Koester, D., Hagen, H. J., et al. 1998, A\&A, 338, 563

Karl, C. A., Napiwotzki, R., Heber, U., et al. 2005, A\&A, 434, 637

Koester, D., Dreizler, S., Weidemann, V., \& Allard, N. F. 1998, A\&A, 338, 612

Koester, D., \& Herrero, A. 1988, ApJ, 332, 910

Koester, D., Napiwotzki, R., Christlieb, N., et al. 2001, A\&A, 378, 556

Koester, D., Rollenhagen, K., Napiwotzki, R., et al. 2005, A\&A, 432, 1025

Koester, D., \& Wolff, B. 2000, A\&A, 357, 587

Langer, N. 1998, A\&A, 329, 551

Langer, N., Heger, A., \& Fliegner, J. 1998, in Fundamental Stellar Properties, IAU Symp., 189, 343

Langer, N., Heger, A., Wellstein, S., \& Herwig, F. 1999, A\&A, 346, L37

Maeder, A., \& Meynet, G. 2000, ARA\&A, 38, 143

Meynet, G., \& Maeder, A. 1997, A\&A, 321, 465

Milkey, R. W., \& Pilachowski, C. A. 1985, PASP, 97, 634

Napiwotzki, R., Christlieb, N., Drechsel, H., et al. 2001, Astron. Nachr., 322, 411

Napiwotzki, R., Christlieb, N., Drechsel, H., et al. 2003, The Messenger, 112, 25

Pilachowski, C. A., \& Milkey, R. W. 1984, PASP, 96, 821

Pilachowski, C. A., \& Milkey, R. W. 1987, PASP, 99, 836
Pruin, K. 2005, Diploma Thesis University of Kiel

Rollenhagen, K. 2004, Diploma Thesis University of Kiel

Schmidt, G. D., \& Norsworthy, J. E. 1991, ApJ, 366, 270

Schmidt, G. D., \& Smith, P. S. 1995, ApJ, 448, 305

Spiegel, E. A., \& Zahn, J.-P. 1970, Comments on Astrophysics and Space Physics, 2, 178

Spruit, H. C. 1998, A\&A, 333, 603

Spruit, H. C. 2002, A\&A, 381, 923

Talon, S., \& Charbonnel, C. 2003, A\&A, 405, 1025

Talon, S., \& Charbonnel, C. 2004, A\&A, 418, 1051

Talon, S., Kumar, P., \& Zahn, J. 2002, ApJ, 574, L175

Thompson, S. E., Clemens, J. C., van Kerkwijk, M. H., \& Koester, D. 2003, ApJ, 589, 921

Unsöld, A. 1968, Physik der Sternatmosphären (Berlin: SpringerVerlag)

van Kerkwijk, M. H., Clemens, J. C., \& Wu, Y. 2000, MNRAS, 314, 209

Weidemann, V. 2000, A\&A, 363, 647

Zahn, J.-P. 1974, in Stellar Instability and Evolution, IAU Symp., 59, 185

Zahn, J. P. 1975, Mémoires of the Société Royale des Sciences de Liège, 8,31

Zahn, J.-P. 1992, A\&A, 265, 115

Zuckerman, B., Koester, D., Reid, I. N., \& Hünsch, M. 2003, ApJ, 596, 477

Zuckerman, B., \& Reid, I. N. 1998, ApJ, 505, L143 